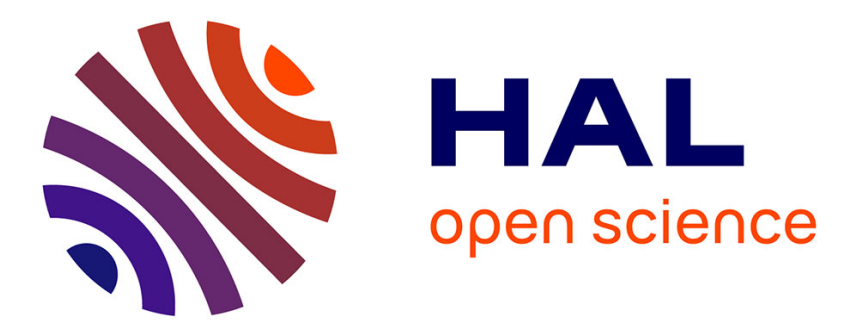

\title{
Model updating techniques for damage detection in concrete beam using optical fiber strain measurement device
}

Julien Waeytens, Bojana Rosić, Pierre-Etienne Charbonnel, Erick Merliot, Dominique Siegert, Xavier Chapeleau, Roland Vidal, Véronique Le Corvec, Louis-Marie Cottineau

\section{To cite this version:}

Julien Waeytens, Bojana Rosić, Pierre-Etienne Charbonnel, Erick Merliot, Dominique Siegert, et al.. Model updating techniques for damage detection in concrete beam using optical fiber strain measurement device. Engineering Structures, 2016, 10.1016/j.engstruct.2016.08.004 . hal-01379214

\author{
HAL Id: hal-01379214 \\ https://hal.inria.fr/hal-01379214
}

Submitted on 16 Dec 2016

HAL is a multi-disciplinary open access archive for the deposit and dissemination of scientific research documents, whether they are published or not. The documents may come from teaching and research institutions in France or abroad, or from public or private research centers.
L'archive ouverte pluridisciplinaire HAL, est destinée au dépôt et à la diffusion de documents scientifiques de niveau recherche, publiés ou non, émanant des établissements d'enseignement et de recherche français ou étrangers, des laboratoires publics ou privés. 


\title{
Model updating techniques for damage detection in concrete beam using optical fiber strain measurement device
}

\author{
J. Waeytens ${ }^{\mathrm{a}, *}$, B. Rosić ${ }^{\mathrm{b}}$, P.-E. Charbonnel ${ }^{\mathrm{c}}$, E. Merliot $^{\mathrm{a}}$, D. Siegert ${ }^{\mathrm{a}}$, X.

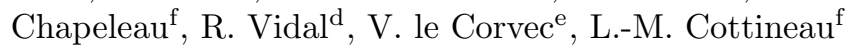 \\ ${ }^{a}$ Université Paris-Est, IFSTTAR \\ Cité Descartes, 14-20 Boulevard Newton, F-77447 Marne-la-Vallée, France \\ ${ }^{b}$ Institute of Scientific Computing, Technische Universität Braunschweig \\ D-38106 Braunschweig, Germany \\ ${ }^{c} C E A, D E N$, DANS, DM2S, SEMT, Laboratoire d'Etude en Mécanique Sismique \\ F-91191 Gif-sur-Yvette, France \\ ${ }^{d}$ ADVITAM \\ 280 Avenue Napoléon Bonaparte, F-92506 Rueil Malmaison, France \\ ${ }^{e}$ Numerical Engineering 85 Consulting Services \\ 196 rue Houdan, F-92330 Sceaux, France \\ ${ }^{f}$ LUNAM Université, IFSTTAR \\ F-44344 Bouguenais, France
}

\begin{abstract}
The article aims at detecting and quantifying early structural damages using deterministic and probabilistic model updating techniques. To achieve this purpose, local information in a form of optical strain measurement is employed. The strategy consists in updating physical parameters associated to damages, such as Young's modulus, in order to minimize the gap between the numerical strain obtained from finite element solves and the strain sensor outputs. Generally, the damage estimation is an ill-posed inverse problem, and hence requires regularization. Herein, three model updating techniques are considered involving different type of regularization: classical Tikhonov regularization, Constitutive Relation Error based updating method and Bayesian approach.

An illustration of these three approaches is proposed for localizing and quantifying an early damage in a real 8 meter post-tensioned concrete beam. Numerical results show that all the methods properly localize the damaged area
\end{abstract}

\footnotetext{
*Corresponding author

Email address: julien.waeytens@ifsttar.fr (J. Waeytens)
} 
and give similar estimation of the damage level.

Keywords: Structural Health Monitoring, damage detection techniques, model updating, optic fiber

\section{Introduction}

Structural Health Monitoring (SHM) has become of crucial importance in the civil engineering community for detecting and localizing damages, reducing the maintenance cost of structures and ensuring the safety of individuals. Three

5 levels of damage assessment [1] can be distinguished: damage detection (level 1), damage localization (level 2) and damage quantification (level 3). Levels 1 and 2 mostly rely on data-driven methods for computing damage indicators from measurements performed on vibrating structures. The damage indicators are generally designed to be sensitive to the variations of the modal signature of the structure under study and at least require a comparison with a reference state; different methods coexist and operate in time or frequency frameworks with deterministic or stochastic inputs. Basics and classical references on SHM can be found in [2, 3], whereas more recent works on vibration-based damage detection and localization are presented in [4, 5] and the references therein. Model updating in turn or model calibration techniques directly allows damage assessment up to level 3. The starting point of these methods is to consider a parametric model (generally derived by using a Finite Element framework) as sufficiently accurate to stand for the behavior of the real structure in an undamaged configuration; this reference model is then compared to the measurements performed on the actual damaged structure, and optimal parameters sets are sought as those ensure the best compatibility between model and measurements. The basic principle behind this consists in assuming that localized structural damage results in a local diminution of stiffness. Over the last few decades, uncertainty quantification or inverse problem resolution has gained substantially in interest and has led to a huge amount of approaches and strategies in various scientific domains. Among the most popular approaches for uncertainty quantification, 
one can non-exhaustively cite : the deterministic parametric residual minimization (deterministic approach, see e.g. [6] and the references therein, or [7, 8] in linear mechanics), the probabilistic Bayesian approach (see e.g. 9] or [10]), and a non-probabilistic Fuzzy approach (see references in [11, 12, 13]). Bayesian approach was previously considered in a two-stage damage detection process consisting of Bayesian and immune genetic algorithms to determine the reduction of Young's moduli [14]. Similarly, in [15, 16] is considered the structural model selection and damage identification given measured data in Monte Carlo like way. However, most of these approaches utilize the expensive numerical algorithms which can be applied only for small dimensional problems. For further mention of existing methodologies on uncertainty quantification and damage assessment, the interested reader is referred to the rich review [17. This paper will focus on three of the existing methods: two of them from the deterministic 40 parametric residual minimization approach and one from the Bayesian inference. In this article the new Bayesian technique following the ideas presented in 18 is suggested to be used for high dimensional problems due to its small computational cost. The method can fully employ linear algebra apparatus and hence can re-use existing fast linear algebra computational libraries.

Post-tensioned concrete beams are widely used in civil engineering structures, in particular for independent span viaducts with prestessed beams. Due to this reason in the French research project named "SIPRIS", an 8 meter post-tensioned concrete beam is constructed and instrumented to simulate the damage scenario and repair actions in a laboratory surrounding. To study and to compare damage detection techniques, the post-tensioned concrete beam is instrumented by a large number of devices: accelerometers, inclinometers, displacement sensors, extensometers, and optic fibers placed along longitudinal reinforcement and groove in the concrete. Herein, we focus on the strain mea55 surement provided by optic fibers. Combining model updating techniques and the local strain information, the aim of this paper is at detecting, localizing and quantifying early damages with the help of deterministic and probabilistic 
numerical procedures. For this purpose three model updating techniques are discussed and compared naming: classical Tikhonov regularization [19], Constitutive Relation Error (CRE) based updating method [20, 21, 22, 23] and Bayesian framework [10, 18. They are analyzed with respect to the measure of the information gain obtained after the updating procedure. In this paper both deterministic and probabilistic approaches are assuming that the real value of the damage parameter is deterministic and give the estimate of its expected value given data. In the latter case the Bayesian posterior distribution represents the confidence bound on the estimate and may encounter uncertainties such as modelling and measurement errors. However, these uncertainties are not quantified per se as for this task further generalizations of the problem have to be provided, and these are not the subject of this paper.

The paper is organized as follows: in Section 2, the considered model updating techniques are summarized and in Section 3 the damage detection results are compared on a real 8 meter post-tensioned concrete beam.

\section{Outlines of model updating techniques for damage detection}

A classical damage theory [24] takes into consideration structural damages via loss of Young's modulus. By assuming the mechanical behavior of the structure to be the static linear elasticity, we further employ the model updating strategies in order to determine a cartography of the Young's modulus, and thus to localize and to quantify its variations due to damage processes. Herein, the potential damaged area is discretized using a coarse mesh " $\mathrm{H}$ " and the Young's modulus is considered piecewise constant on the elements. The corresponding vector of Young's modulus to be updated is denoted by $\mathbf{E}$. On the other side, a more refined mesh " $\mathrm{h}$ " is used to solve the mechanical problem by the Finite Element Method

$$
\mathbb{K}(\mathbf{E}) \mathbf{U}=\mathbf{F}
$$


in which the nodal displacement $\mathbf{U}$ is equal to prescribed displacement $\mathbf{U}_{\mathbf{d}}$ on the kinematic boundary. In the finite element setting the rigidity matrix $\mathbb{K}$ (symmetric and positive definite) is parameterized by a vector $\mathbf{E}$, whereas the vector $\mathbf{U}$ (resp. F) is associated to the nodal values of the displacement (resp. the static loading). To this equation, one may add the mathematical prediction of the sensor outputs $\epsilon^{\operatorname{sim}}=\mathbb{B} \mathbf{U}$, in which $\mathbb{B}$ is a strain-displacement matrix extracting the numerical strain at the sensor location and $\mathbf{U}$ is the solution of Eq. (1).

The numerical solution $\epsilon^{\text {sim }}$ compared to the sensor outputs $\epsilon^{\text {mes }}$ gives the so-called model error assumed to be of much higher magnitude than the measurement error. In order to neutralize the gap between the numerical solution and the sensor outputs, we seek the optimal value of the vector $\mathbf{E}$. This further coincides with an early damage detection given the local information on strain measurements. The process of estimating $\mathbf{E}$ given noisy measurement data is generally ill-posed [6], and hence requires regularization. This can be achieved in many different ways, three of which are considered in this paper. In the following sub-sections, the three approaches for regularizing the ill-posed inverse problem are discussed and presented.

\subsection{Tikhonov-based technique}

Damage localization and quantification can be formulated as a constrained minimization problem. The following approach has been proposed in [21]. We seek the vector $\mathbf{E}$ of Young modulus which minimizes the functional

$$
J_{T}(\mathbf{E})=\frac{1}{2}\left(\mathbb{B} \mathbf{U}-\epsilon^{\mathbf{m e s}}\right)^{T}\left(\mathbb{B} \mathbf{U}-\epsilon^{\mathbf{m e s}}\right)+\frac{\alpha_{T}}{2} s_{T}\left(\mathbf{E}-\mathbf{E}_{\mathbf{u d}}\right)^{T}\left(\mathbf{E}-\mathbf{E}_{\mathbf{u d}}\right)
$$

under the equilibrium constraint (i.e. the nodal displacements have to verify the mechanical problem given in Eq. (1)).

In Eq. (2) the first term of the functional $J_{T}$ measures the gap between the numerical solution and the sensor outputs, whereas the second term corresponds to a Tikhonov regularization [19]. The role of the latter one is to enforce the Young's modulus $E$ to be in the vicinity of the undamaged Young modulus $E_{u d}$, 
and thus to avoid the case of having several unphysical minima. For a physical homogeneity of the cost function terms and customization of their weights, the parameter $s_{T}$ and the normalized ponderation coefficient $\alpha_{T}$ are introduced, respectively. The numerical value of the last one is determined according to the discrepancy principle due to Morozov [25]. In practice, $\alpha_{T}$ is chosen such that the maximal value of the data misfit at the end of the model updating process is close to the measurement error and strictly superior. Commonly, to solve a constrained minimization problem in Eq. (2), one introduces the Lagrange multiplier $\boldsymbol{\Psi}$ and studies the Lagrangian function

$$
\mathcal{L}_{T}(\mathbf{E}, \mathbf{\Psi})=J_{T}(\mathbf{E})-\Psi^{T}(\mathbb{K}(\mathbf{E}) \mathbf{U}-\mathbf{F})
$$

Writing the stationarity of the Lagrangian with respect to $\boldsymbol{\Psi}$ and $\mathbf{U}$ leads respectively to the discrete equilibrium equation (1) and to the so-called mechanical adjoint problem

$$
\mathbb{K} \boldsymbol{\Psi}=\mathbb{B}^{T}\left(\mathbb{B} \mathbf{U}-\epsilon^{\text {mes }}\right)
$$

Note that the adjoint problem is quite similar to the direct problem in Eq. (1). In both problems, the rigidity matrix and the boundary associated to the kinematic conditions are the same. Nevertheless, the specificity of the adjoint problem lies in the following facts: the kinematic conditions vanish, the loading is located at the sensor position and its amplitude is given by the data misfit. The third stationary condition is obtained by differentiating the Lagrangian with respect to the Young's modulus, which further gives us the gradient of the functional $J_{T}$

$$
\nabla \mathbf{J}_{\mathbf{T}}=\left\{\begin{array}{c}
\frac{\partial J}{\partial E_{1}}=-\boldsymbol{\Psi}^{T} \frac{\partial \mathbb{K}}{\partial E_{1}} \mathbf{U}+\alpha_{T} s_{T}\left(E_{1}-E_{u d}\right) \\
\cdots \\
\frac{\partial J}{\partial E_{n E}}=-\Psi^{T} \frac{\partial \mathbb{K}}{\partial E_{n E}} \mathbf{U}+\alpha_{T} s_{T}\left(E_{n E}-E_{u d}\right)
\end{array}\right\}
$$

Its form simplifies in the linear elastic isotropic case for which some of the 
appearing partial derivatives read

$$
\begin{aligned}
\boldsymbol{\Psi}^{T} \frac{\partial \mathbb{K}}{\partial E_{i}} \mathbf{U} & =\frac{\partial \lambda}{\partial E_{i}} \int_{\Omega_{i}} \operatorname{div}(\boldsymbol{\psi}) \operatorname{div}(\mathbf{u}) d \Omega+\frac{\partial \mu}{\partial E_{i}} \int_{\Omega_{i}} \operatorname{Tr}[\epsilon(\boldsymbol{\psi}) \epsilon(\mathbf{u})] d \Omega \\
\text { with } \frac{\partial \lambda}{\partial E_{i}} & =\frac{\nu}{(1+\nu)(1-2 \nu)}, \frac{\partial \mu}{\partial E_{i}}=\frac{1}{2(1+\nu)}
\end{aligned}
$$

In order to compute the functional gradient at a lower computational cost than the straightforward use of the finite-difference schemes the adjoint solution $\Psi$ is employed.

Finally, to localize and to quantify the damage, we seek the vector E of Young's moduli minimizing the functional $J_{T}$ defined in Eq. (2). The minimization problem is solved in an iterative way by means of the steepest descent method. At each iteration, the steps are the following:

1. Solve the direct problem (1) considering the vector $\mathbf{E}_{\text {old }}$ of Young's moduli from the previous iteration, and get $\mathbf{U}$;

2. Solve the adjoint problem (4) and get $\boldsymbol{\Psi}$;

3. Compute the descent direction, i.e. the functional gradient $\nabla \mathbf{J}_{\mathbf{T}}$ using Eq. (5) and (6), and determine the descent step $\beta$ by a linesearch approach;

4. Update the vector $\mathbf{E}$ of Young's moduli: $\mathbf{E}_{\mathbf{n e w}}=\mathbf{E}_{\mathbf{o l d}}-\beta \nabla \mathbf{J}_{\mathbf{T}}$.

\subsection{Constitutive Relation Error technique}

The concept of the constitutive relation error was initially introduced for the estimation of the discretization error in the finite element simulations [26]. Then, it has been extended to model updating purposes [8, 20, 22, 27, 28, This framework is particularly adapted for the determination of the constitutive relation parameters, such as Young's moduli. In this technique, we distinguish a nodal displacement $\mathbf{U}$ satisfying the kinematic boundary conditions (7) and a nodal displacement $\mathbf{V}$ verifying the equilibrium equations in a finite element sense (8)

$$
\begin{aligned}
\mathbb{C} \mathbf{U} & =\mathbf{U}_{\mathbf{d}} \\
\mathbb{K}(\mathbf{E}) \mathbf{V} & =\mathbf{F}
\end{aligned}
$$


where $\mathbb{C} \mathbf{U}$ represents the extraction of the nodal displacement on the kinematic boundary with prescribed displacement $\mathbf{U}_{\mathbf{d}}$.

The constitutive relation parameters, i.e. the vector $\mathbf{E}$ of Young's moduli, are determined by acquiring the energy gap between the kinematic field $\mathbf{U}$ and the static field $\mathbf{V}$ together with the misfit between the measurements to be minimized. The corresponding functional is called the "modified constitutive relation error", and is defined by

$J_{C R E}(\mathbf{E})=\frac{\alpha_{C R E}}{2} s_{C R E}\left(\mathbb{B} \mathbf{U}-\epsilon^{\mathbf{m e s}}\right)^{T}\left(\mathbb{B} \mathbf{U}-\epsilon^{\mathbf{m e s}}\right)+\frac{1}{2}(\mathbf{U}-\mathbf{V})^{T} \mathbb{K}(\mathbf{E})(\mathbf{U}-\mathbf{V})$

The first term in Eq. (9) corresponds to the data misfit, as in the Tikhonovbased method, whereas the second term is associated to the constitutive relation error. The parameter $s_{C R E}$ ensures the physical homogeneity of both terms in the functional (9). As in Section 2.1 the normalized ponderation coefficient $\alpha_{C R E}$ is determined using the discrepancy principle.

To solve the minimization problem, we introduce the Lagrangian given by:

$$
\mathcal{L}_{C R E}(\mathbf{E}, \boldsymbol{\Psi})=J_{C R E}(\mathbf{E})-\Psi^{T}(\mathbb{K}(\mathbf{E}) \mathbf{V}-\mathbf{F})
$$

Finding its stationary points leads to the following system:

$$
\begin{aligned}
& \mathbb{K}(\mathbf{U}-\mathbf{V})+\alpha_{C R E} s_{C R E} \mathbb{B}^{T}\left(\mathbb{B} \mathbf{U}-\epsilon^{\text {mes }}\right)=\mathbf{0} \\
& \mathbb{K}(\mathbf{U}-\mathbf{V})+\mathbb{K} \mathbf{\Psi}=0 \\
& \mathbb{K} \mathbf{V}=\mathbf{F}
\end{aligned}
$$

Hence the kinematically admissible field $\mathbf{U}$, the statically admissible field $\mathbf{V}$ and the adjoint field $\boldsymbol{\Psi}$ are obtained solving:

$$
\begin{aligned}
& \left(\mathbb{K}+\alpha_{C R E} s_{C R E} \mathbb{B}^{T} \mathbb{B}\right) \mathbf{U}=\mathbf{F}+\alpha_{C R E} s_{C R E} \mathbb{B}^{T} \epsilon^{\text {mes }} \\
& \mathbb{K} \mathbf{V}=\mathbf{F} \\
& \mathbf{\Psi}=\mathbf{V}-\mathbf{U}
\end{aligned}
$$



admissible field $\mathbf{U}$ and the statically admissible field $\mathbf{V}$ are equals, then the data misfit vanishes, i.e. $\mathbb{B} \mathbf{U}-\epsilon^{\text {mes }}=\mathbf{0}$.

Contrary to the previous method, herein the model parameters to be updated are chosen according to a constitutive relation error criteria instead of the functional gradient. First, we evaluate the contribution denoted $\varepsilon_{C R E}^{i}$ of each subdomain $\Omega_{i}$ (associated to H-size mesh), whose Young's modulus corresponds to $E_{i}$, to the constitutive relation error term, involved in Eq. (9).

$$
\varepsilon_{C R E}^{i}=\frac{1}{2}\left(\mathbf{U}_{\mathbf{i}}-\mathbf{V}_{\mathbf{i}}\right)^{T} \mathbb{K}_{i}\left(E_{i}\right)\left(\mathbf{U}_{\mathbf{i}}-\mathbf{V}_{\mathbf{i}}\right)
$$

where $\mathbf{U}_{\mathbf{i}}, \mathbf{V}_{\mathbf{i}}$, and $\mathbb{K}_{i}$ are the restrictions of $\mathbf{U}, \mathbf{V}$ and $\mathbb{K}$ on the subdomain

Then, we deduce the highest constitutive relation error $\varepsilon_{C R E}^{\max }=\max _{i} \varepsilon_{C R E}^{i}$. The model parameter $E_{i}$ associated to the subdomain $\Omega_{i}$ is updated if $\varepsilon_{C R E}^{i}>$ $0.8 \varepsilon_{C R E}^{\max }$. The selection of the most erroneous parameters associated to the highest CRE contribution is called localization step and in practice, for each iteration, only one or two constitutive parameters are corrected. The regularization of the ill-posed inverse problem is indeed permitted by the alternating localization/correction step sequence that is adopted here.

To summarize, the determination of the vector $\mathbf{E}$ of Young's moduli minimizing the functional $J_{C R E}$, defined in Eq. (9), is achieved iteratively. At each iteration, the steps are the following:

1. Consider the vector $\mathbf{E}_{\text {old }}$ of Young's moduli from the previous iteration;

2. Determine the kinematically admissible field $\mathbf{U}$ solving the first equation in 12 ;

3. Determine the statically admissible field $\mathbf{V}$ solving the second equation in 145 (12);

4. Evaluate the constitutive relation error $\varepsilon_{C R E}^{i}$ from Eq. 13 in each potential damaged subdomain $\Omega_{i}$ and get the highest constitutive relation 
error $\varepsilon_{C R E}^{\max }$;

5. Update only the model parameters $E_{i}$ verifying $\varepsilon_{C R E}^{i}>0.8 \varepsilon_{C R E}^{\max }$. If only one model parameter is selected, a standard linesearch is employed to minimize the functional $J_{C R E}$. Otherwise, the steepest descent method is applied.

6. Deduce the new vector $\mathbf{E}_{\text {new }}$ of Young's moduli.

\subsection{Bayesian technique}

The previously described Tikhonov and Constitutive Relation Error regularization procedures mathematically promote the idea of loss functions which measure the error in predicting the observation data in a purely deterministic way. However, the estimation of Young's moduli E, i.e. localization of damage, can be considered in another setting which allows the prior expert beliefs on

unknown parameters to enter the model. Prior beliefs describe the quantity of matter before gaining any evidence, and are commonly described by a probability density function $p(\mathbf{E})$. This function plays the regularization role for an inverse problem considered in a Bayesian setting

$$
\pi(\mathbf{E} \mid \mathbf{z})=\operatorname{const} L(\mathbf{z} \mid \mathbf{E}) p(\mathbf{E})
$$

In this manner the process of damage localization becomes well posed and relies on the computation of the probability density function $\pi(\mathbf{E} \mid \mathbf{z})$ that best fits the data $\mathbf{z}:=\epsilon^{\mathbf{m e s}}$. However, one may note that the process of updating the prior belief $p(\mathbf{E})$ to a more realistic description $\pi(\mathbf{E} \mid \mathbf{z})$, called posterior, comes on the expense of solving a probabilistic problem. Namely, $p(\mathbf{E})$ is corrected by the information gain $L(\mathbf{z} \mid \mathbf{E})$ describing of how likely the measurement data are given the model. The last one is essentially the measure of distance $\mathbf{z}-\hat{\mathbf{z}}$, e.g. for normally distributed likelihood one has $L(\mathbf{z} \mid \mathbf{E})=N\left(\mathbf{z}-\hat{\mathbf{z}}, C_{\epsilon}\right)$, which implicitly includes the prediction of the measurement data $\hat{\mathbf{z}}$ given $\mathbf{E}$. 
Following the derivation in [10, 18, one may rewrite the Bayes rule in terms of quadratic minimization

$$
\mathbf{E}_{\mathbf{a}}=\min _{\varphi \in \Phi}\|\mathbf{E}-w(\varphi, \mathbf{E})\|^{2}
$$

in which the space $\Phi$ spans the set of all measurable functions $w(\varphi, \mathbf{E}):=$ $\varphi(\mathbf{z}(\mathbf{E}))$. As the complete space $\Phi$ cannot be represented by computations, the previous minimization is further relaxed to

$$
\mathbf{E}_{\mathbf{a}}=\min _{\varphi \in \Phi^{n}}\|\mathbf{E}-w(\varphi, \mathbf{E})\|^{2}
$$

in which the closed subspace $\Phi^{n} \in \Phi$ is spanned by polynomials of up to 180 n-th degree

$$
\varphi(\mathbf{z})=\mathbb{H}_{0}+\mathbb{H}_{1} \mathbf{z}+\ldots+\mathbb{H}_{k} \mathbf{z}^{(\otimes k)}+\ldots+\mathbb{H}_{n} \mathbf{z}^{(\otimes n)}
$$

Here, the polynomial coefficients $\mathbb{H}_{k}$ are symmetric and $k$ linear tensors, whereas $\mathbf{z}^{(\otimes k)}$ is symmetric tensor product of $\mathbf{z}$ 's taken $k$ times with itself. Satisfying the stationary condition of Eq. (16) one obtains the linear system of equations

$$
\left(\begin{array}{cccc}
1 & <z> & \ldots & <z^{\otimes n}> \\
<z> & <z^{\otimes 2}> & \ldots & <z^{\otimes(n+1)}> \\
\ldots & \ldots & \ldots & \ldots \\
<z^{\otimes n}> & <z^{\otimes(n+1)}> & \ldots & <z^{\otimes 2 n}>
\end{array}\right)\left(\begin{array}{c}
\mathbb{H}_{0} \\
\mathbb{H}_{1} \\
\ldots \\
\mathbb{H}_{n}
\end{array}\right)=\left(\begin{array}{c}
<\mathbf{E}> \\
<\mathbf{E} \otimes \mathbf{z}> \\
\ldots \\
<\mathbf{E} \otimes \mathbf{z}^{\otimes n}>
\end{array}\right)
$$

185

in which $<\bullet>$ denotes the mathematical expectation of the quantity of consideration:

$$
<q>=\int_{\Omega} q(\omega) P(d \omega)
$$

i.e. the high dimensional integral over the probability space $\Omega$ with the probability measure $P$. 
In a special case when $n=1$ the system in Eq. (18) reduces to the so-called linear Bayesian formula (see [10])

$$
\mathbf{E}_{\mathbf{a}}=\mathbf{E}_{\mathbf{f}}+\mathbb{H}_{1}(\mathbf{z}-\hat{\mathbf{z}})
$$

in which $\mathbb{H}_{1}$ plays the role of the Kalman gain, and $\mathbf{E}_{\mathbf{a}}$ and $\mathbf{E}_{\mathbf{f}}$ are the posterior and prior random variables, respectively.

Employing the random variables instead of probability density functions, the mathematical formulation given in Eq. (18) offers many more possibilities for the numerical computation than the Bayes rule in Eq. (14). For example, if the random variables $E$ are represented by a set of samples (realizations) then Eq. 207 is equivalent to the well-known ensemble Kalman filter. Otherwise, if the functional approximation approach is used as described in [18] then Eq. 20] reduces to the polynomial chaos based update. The same holds for the higher order update following from Eq. (17) and (18).

In this paper the random variables are represented by a set of samples (realizations) $\mathbf{E}\left(\omega_{1}\right), \ldots, \mathbf{E}\left(\omega_{N}\right)$ such that the system matrix and right hand side in Eq. 18 are easily evaluated. In this manner the computation of the posterior via Eq. (17) becomes rather simple. This kind of approach is similar to the ensemble Kalman filter, and hence favorable for the damage detection where the computation of the predicted measurement is difficult and expensive.

210

To summarize, the determination of the vector $\mathbf{E}$ of Young's moduli in a probabilistic manner is done in several steps:

1. Choose the prior distributions for the vector of Young's moduli;

2. Represent random variables $\mathbf{E}(\omega)$ by a set of samples $\mathbf{E}\left(\omega_{1}\right), \ldots, \mathbf{E}\left(\omega_{N}\right)$;

3. Predict the measurement data $\hat{\mathbf{z}}\left(\omega_{1}\right), \ldots, \hat{\mathbf{z}}\left(\omega_{N}\right)$ given $\mathbf{E}\left(\omega_{1}\right), \ldots, \mathbf{E}\left(\omega_{N}\right)$;

4. Solve the system in Eq. 18;; 
5. Make the estimation of posterior by using Eq. (17), i.e.:

$$
\mathbf{E}_{\mathbf{a}}=\mathbf{E}_{\mathbf{f}}+\mathbb{H}_{1}(\mathbf{z}-\hat{\mathbf{z}})+\mathbb{H}_{2}\left(\mathbf{z}^{\otimes 2}-\hat{\mathbf{z}}^{\otimes 2}\right)+\text { h.o.t }
$$

Note that in the previous equation the measurement error $\varepsilon_{1}$ as well as possible modelling errors $\varepsilon_{2}$ are taken into account. Namely, the predicted measurement $\hat{z}$ is modelled as $\hat{z}=z_{f}+\varepsilon$ in which $z_{f}$ is the prediction of the structural response given uncertain Young's moduli $E_{f}$, whereas $\varepsilon$ is independent uncertain term encountering the data-model discrepancies in a following manner $\varepsilon=\varepsilon_{1}+\varepsilon_{2}$. The distribution of $\varepsilon$ is taken a priori (e.g. normally distributed), but later can be updated in a similar manner as shown previously. The main goal of this paper is not to quantify the existing discrepancies (i.e. their distribution), but to estimate the deterministic value of the Young's modulus and its confidence bounds given structural model. In order to identify aleratoric and epistemic uncertainties, one has to pose different kind of question than the one posed here. For more information the reader is addressed to e.g. [29, 30]

3. Comparison of damage detection results on a 8 meter post-tensioned concrete beam

\subsection{Description of the static test on the 8 meter post-tensioned concrete beam}

Let us consider a 8 meter post-tensioned concrete beam represented in Figure 1 which is simply supported on two elastomer supports. The length, the height and the depth are $L=8 m, H=0.6 m$ and $e=0.3 m$. A static loading of $10 k N$ is applied at the middle of the beam. Initially, only the two longitudinal cables are activated to prestress the concrete beam. After damaging strongly the structure by means of an overload (higher than $10 k N$ ), the curved cable was used to close the cracks and to repair the beam. In the considered experiments, we focus on the detection of early damage which may not be detected by visual inspection. 

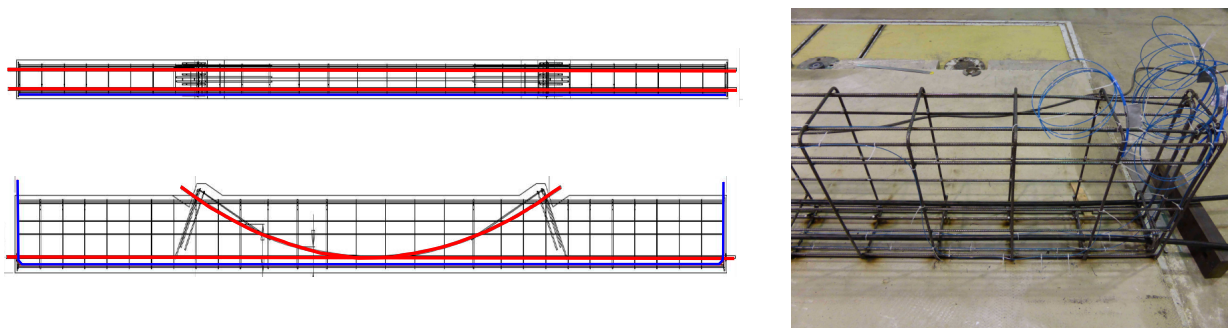

Figure 1: Post-tensioned concrete beam of 8 meter length by means of 3 steel cables (in red)

- Optic fiber (in blue) on a longitudinal armature

\subsection{Strain measurement using optic fiber}

In civil engineering structures, there is a growing interest in fiber optic sensors because they offer interesting benefits when compared with traditional sensors. Their main advantages are small size, electrically passive operation, electromagnetic immunity, flexibility, corrosion resistance, etc. Moreover, fiber optic sensors can be used to perform local or distributed measurements with precision in a wide range of strain and temperature.

For early damage detection, distributed sensing technique is a promising technology because in one acquisition, a lot of measurement points can be obtained along a long length of fiber optic. Two distributed fiber optic sensing techniques, called "Brillouin" and "Rayleigh", are available. The main difference between the two techniques is the spatial resolution. The Rayleigh technique has the highest spatial resolution. Typically, it is $1 \mathrm{~cm}$ for Rayleigh and $0.5-1 \mathrm{~m}$ ( $25 \mathrm{~cm}$ for the best equipment) for Brillouin techniques. This high spatial resolution of measurements is an asset for crack detection. However, the length range is shorter for the Rayleigh technique. It is limited to $70 \mathrm{~m}$ while the length range of the Brillouin technique could be higher than $1 \mathrm{~km}$. Another parameter to take into account is the time of measurement, less than 1minute for the Rayleigh technique and up to 10 -15minutes (depending of the length range and resolution chosen) for the Brillouin technique. For both distributed 
sensing techniques, no dynamic measurements can be obtained but it should not be a limitation since cracking is a long term process of damage. In this study, only the use of the Rayleigh distributed sensing technique with embedded fiber optics in concrete beam was tested for early detection and localization of cracks.

Rayleigh distributed sensing technique is based on the measurement of the backscattered light (Rayleigh signal) produced during the propagation of light waves in the core of a fiber optic. This phenomenon is due to random fluctuations of the refractive index profile along the length of the fiber optic. More precisely, it is based on measurement of the shifts in the local Rayleigh backscatter pattern which depend on strain and temperature. Two successive measurements of the Rayleigh signal are necessary to obtain a strain or temperature 275 profile. The first is used as reference. The typical accuracy of the Rayleigh sensing technique is better than $\pm 1^{\circ} \mathrm{C}$ in temperature and $\pm 5 \mathrm{pm}$ in strain with a centimetric spatial resolution.

In our experiment, an optic fiber (represented in blue in Figure 1) was put along a longitudinal reinforcement at a distance of $30 \mathrm{~mm}$ from the bottom part of the beam. To measure the strain in the concrete beam induced by the static loading, two measurements of Rayleigh signal were necessary. The first measurement (reference) is signal obtained on the unloaded configuration, whereas the second measurement is a signal coming from the configuration loaded by $10 k N$. Note that the room temperature is kept constant during signal Rayleigh measurements, which further allows the temperature effect to be neglected. In Figure 2, the brown curve shows the strain measurement obtained by the fiber optic which is in accordance with a three points bending test as expected. However, due to a crack a small peak appears at $0.7 \mathrm{~m}$ from the middle of the beam. Note that this peak can be detected by the fiber optic because the Rayleigh sensing technique has a high spatial resolution. So, this technique of measurement permits to localize accurately the location of cracks. However, the quantification of cracks (length and opening) is less obvious. Indeed, the strain transfer 


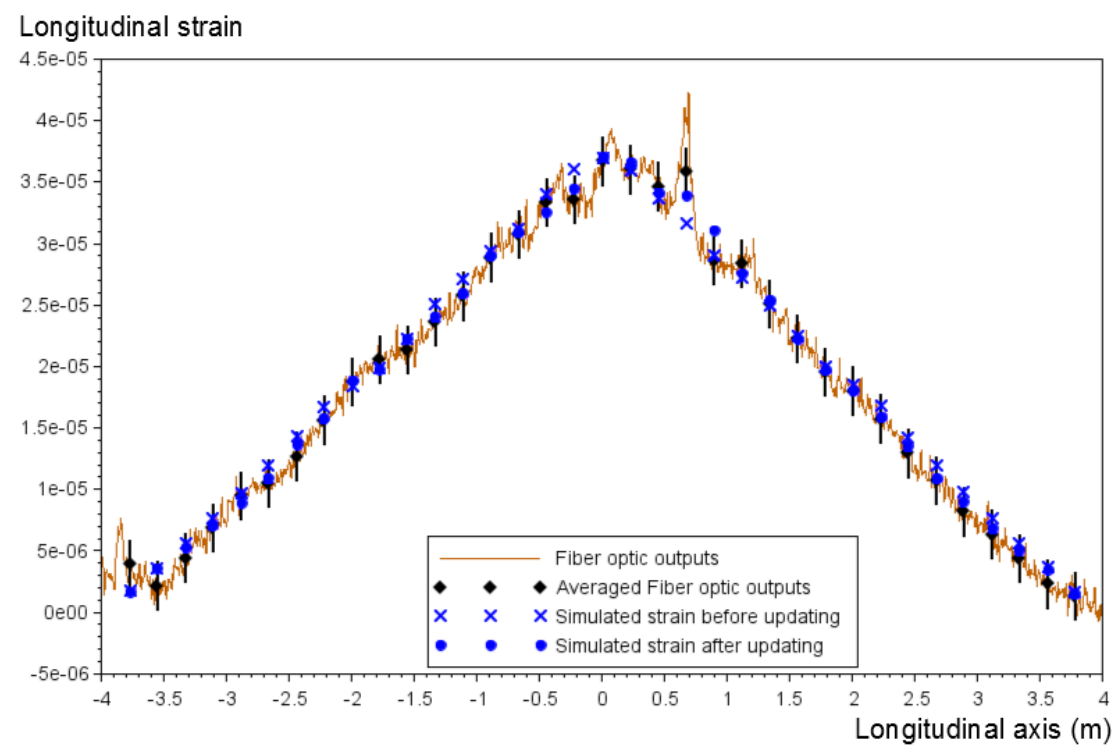

Figure 2: Strain sensor outputs from optic fiber (in brown) and simulated strain before and after Tikhonov-based model updating process

from the tested material to the core of the fiber depends on the structure of the optical cable, and its protective and adhesive layers. Each fiber optic cable has its own mechanical transfer function. For accurate crack opening measurements, it is essential to know the mechanical transfer function. However, its determination is not easy and requires to combine experimental results and numerical modeling. Another approach is to use model updating techniques for damage detection as those presented above (Section 2).

\subsection{Damage detection results}

The goal is to localize and to quantify a potential damage on the 8 meter post-tensioned concrete beam using strain sensor outputs and model updating techniques. Before the updating process, the undamaged Young's modulus $E_{u d}=25 G P a$ is considered in the whole structure. To restrict the number of model parameters to be updated, the potential damaged area, i.e. lower part of the beam, is decomposed into 21 subdomains along the longitudinal axis. We 
aim at updating the Young's modulus in each subdomain. The results obtained from the three different model updating techniques presented in Section 2 are compared. Instead of considering the primary optic fiber outputs (about $1 \mathrm{~cm}$ resolution) as input in all the model updating techniques, we use a $40 \mathrm{~cm}$ averaged of the optic fiber outputs (see black diamond in Figure 2). Hence, we get 35 averaged strain sensor outputs. The measurement error is estimated at $2 \mu \mathrm{m} / \mathrm{m}$.

In Figure 2, we observe that the simulated strain is closed to the strain sensor outputs. The highest data misfit is located at $0.7 \mathrm{~m}$ from the middle of the beam ( $x=0.7$ in Figure 2), which may correspond to a damaged area. In Tikhonovbased model updating, we take $s_{T}=1 /\left(E_{u d}^{2}\right)$ and we find that the normalized ponderation coefficient $\alpha_{T}=2 \times 10^{-10}$ allows the highest data misfit at the end of the model updating process to be closed to the measurement error and strictly superior. The simulated strain after Tikhonov updating is represented in Figure 2 (blue circle) and the updated values of the Young modulus is given in Figure 3. The highest reduction of Young modulus, estimated at 8\%, is located in the subdomain 13. It exactly corresponds to the subdomain with a crack which was brought out by the optic fiber outputs (see Section 3.2).

Concerning the constitutive relation error technique, to verify the discrepancy principle we take $\alpha_{C R E}=4 \times 10^{-7}$ and $s_{C R E}=1 /(L H e)$. In Figure 4 the contribution of the constitutive relation error in each subdomain is represented at the first and the second iteration of the model updating technique. At iteration 0, the highest error $\varepsilon_{C R E}^{\max }$ corresponds to the error in the subdomain 13. The red line is associated to the $0.8 \varepsilon_{C R E}^{\max }$ threshold. This threshold being exceeded only by the error in the subdomain 13, only the Young's modulus in subdomain 13 is updated. The most import decrease of the functional $J_{C R E}$ is achieved at iteration 0 when updating the Young's modulus $E_{13}$. The func335 tional $J_{C R E}$ goes from $1.02 \times 10^{-5}$ to $8.53 \times 10^{-6}$. After iteration 1 , the model updating process should be stopped. Indeed, after iteration 1 the modifications of the Young's moduli and the decreases of the functional are not significant, i.e. few $\mathrm{MPa}$ variation for $\mathrm{E}$ and about $10^{-9}$ variation for $J_{C R E}$. In Figure 


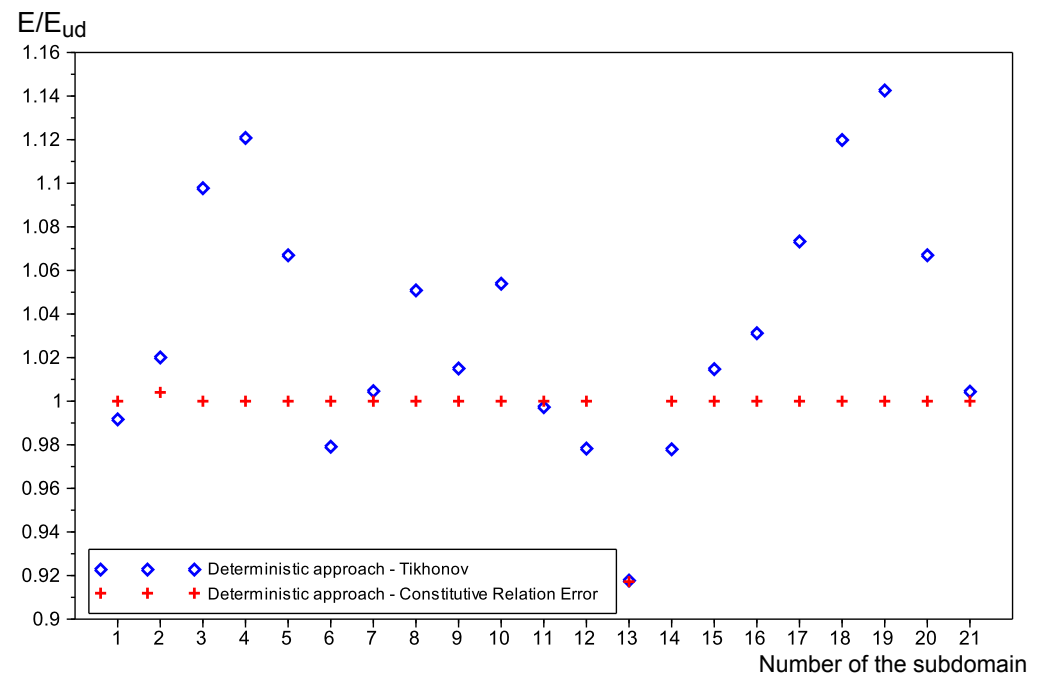

Figure 3: Normalized Young modulus $E / E_{u d}$ in each subdomain after deterministic model updating strategies - Comparison of Tikhonov and Constitutive Relation Error results.

5. the cartography of the updated Young modulus is given. As observed in 340 Figure 3 contrary to the Tikhonov strategy the constitutive relation error technique only updates the Young's modulus in subdomain 13. Lastly, we note that both deterministic methods give the same estimation of the damage level in this subdomain, i.e. $8 \%$ reduction of the Young's modulus.

Finally, the identification of the local damage by approximations of Bayesian inference is undergone by assuming the normalized Young's moduli to be independent and uncorrelated Gaussian random variables with mean equal to 0.9 and standard deviation 0.1, see Figure 6. This choice is taken against logical reason saying that the normal distribution is not a natural choice for the description of the positive defined material properties. However, one may argue that the probability of negative value occurring for small standard deviations compared to the mean value is relatively small. The posterior is estimated using quadratic and linear Bayesian formula and ensemble of 100 samples (i.e. 100 forward simulations). The posterior results are similar to those obtained by the previous two procedures. Namely, the damaged area is appearing to be at 

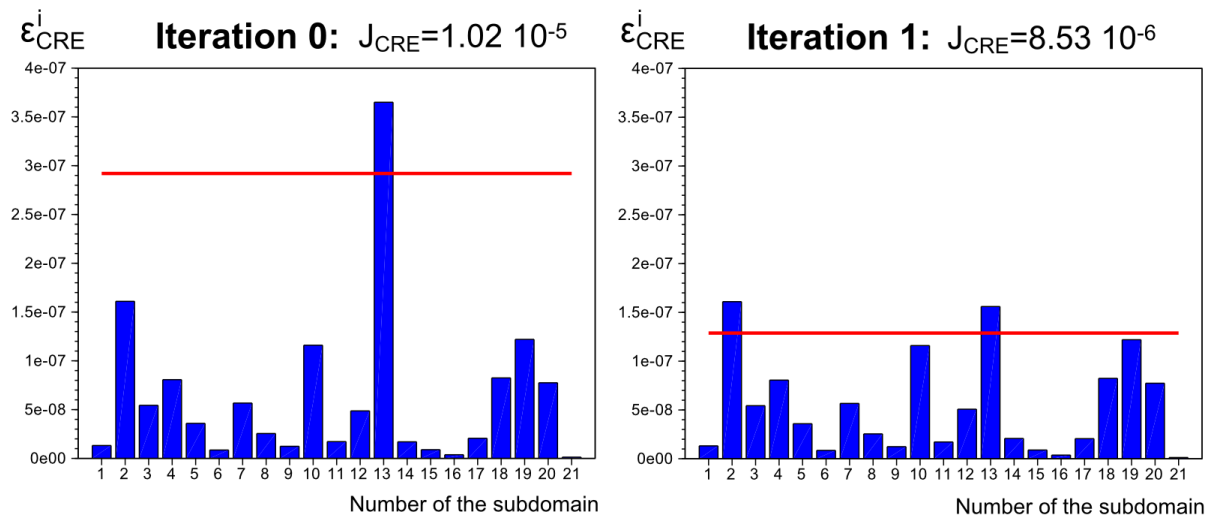

Figure 4: Constitutive Relation Error in each subdomain at iteration 0 (at left) and at iteration 1 (at right) - At iteration 0, Young modulus in subdomain 13 has to be updated.

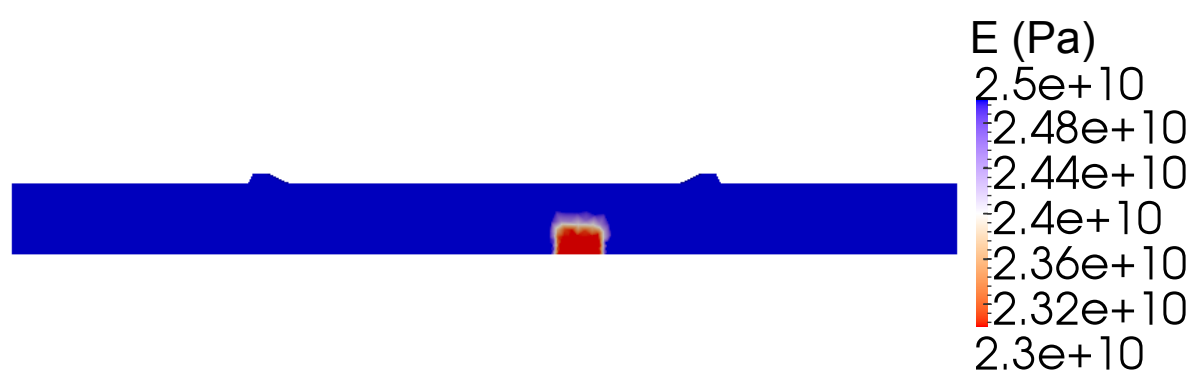

Figure 5: Cartography of Young modulus after Constitutive Relation Error-based model updating process - the damaged area is in red and it corresponds to a $8 \%$ damage. 


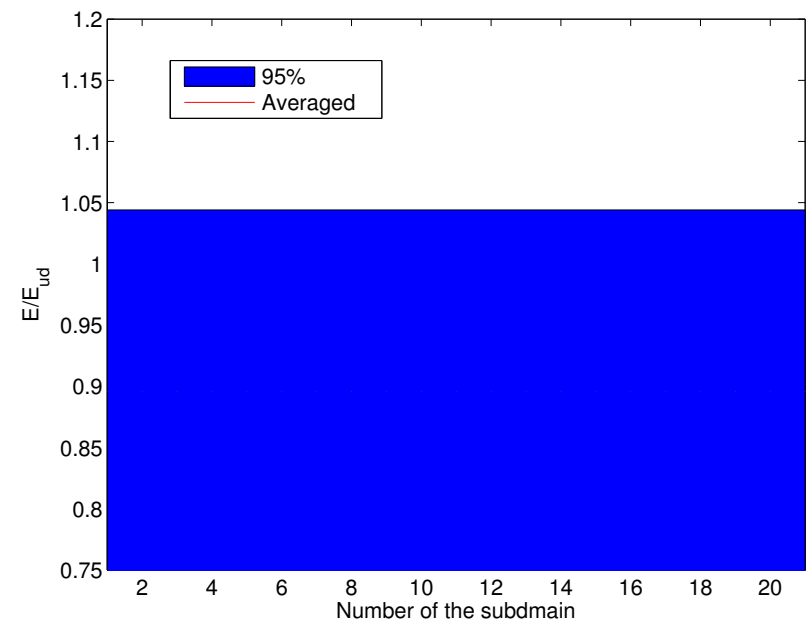

Figure 6: 95\% interval describing prior distribution 


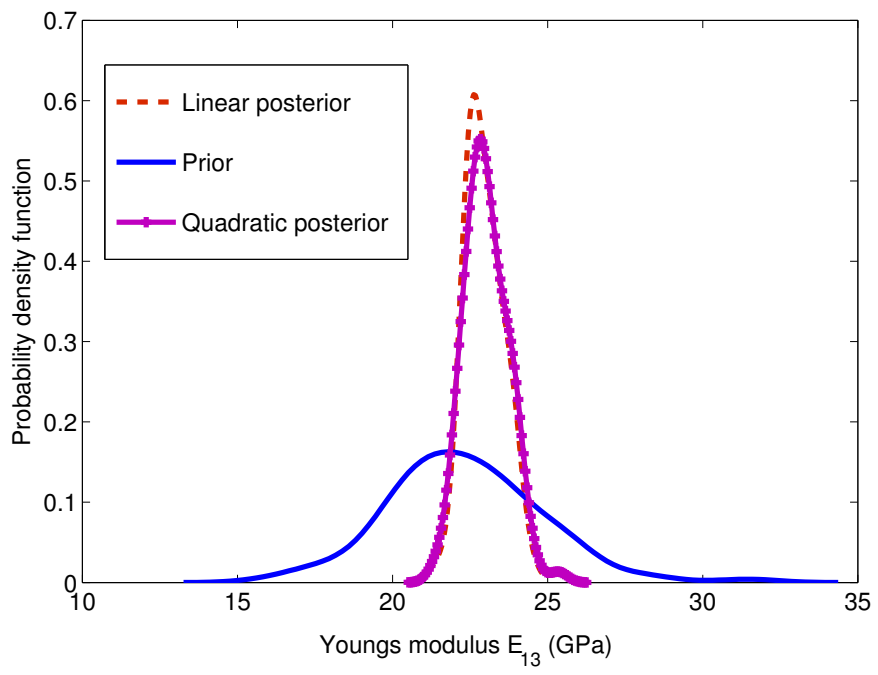

Figure 7: Probability densitiy functions of linear and quadratic Bayesian estimate of Young's modulus in subdomain 13 compared to the prior assumption.

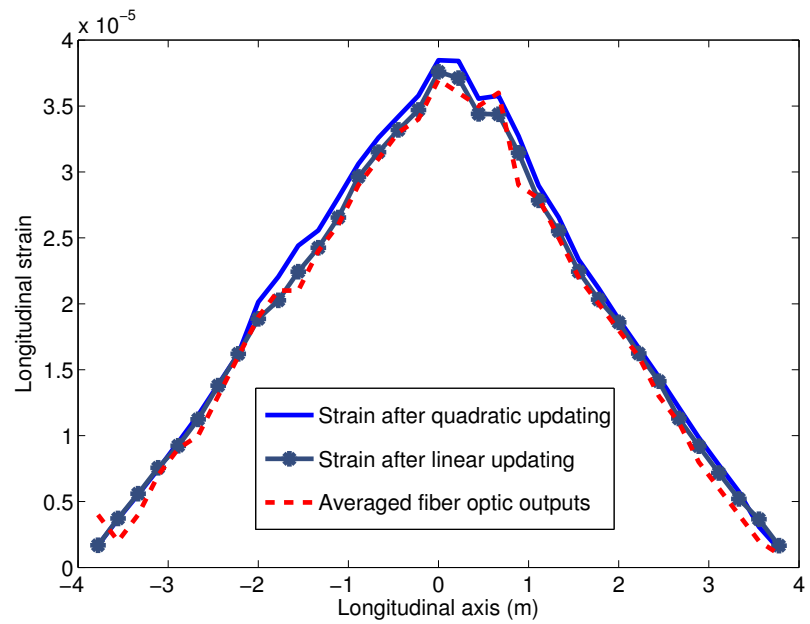

Figure 8: Comparison of posterior modes obtained after linear and quadratic Bayesian update to the observed value of the strain 

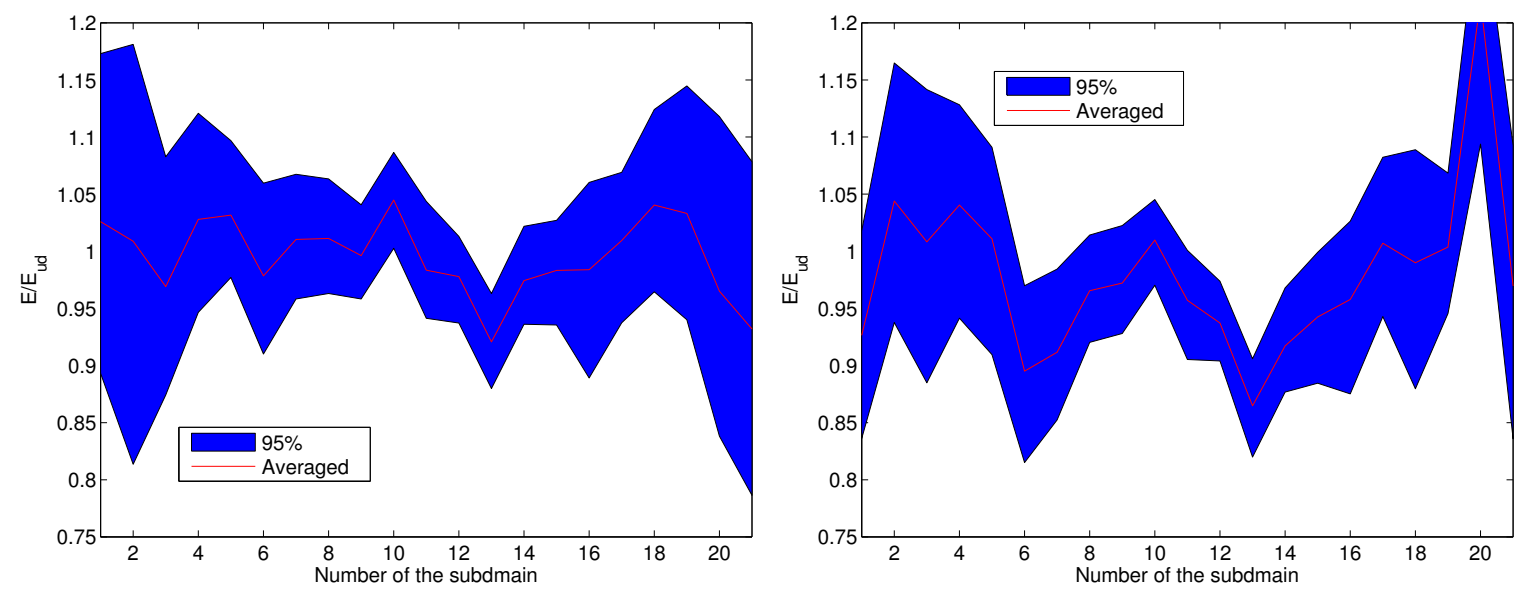

Figure 9: 95\% probability interval describing linear (left) and quadratic (right) posteriors

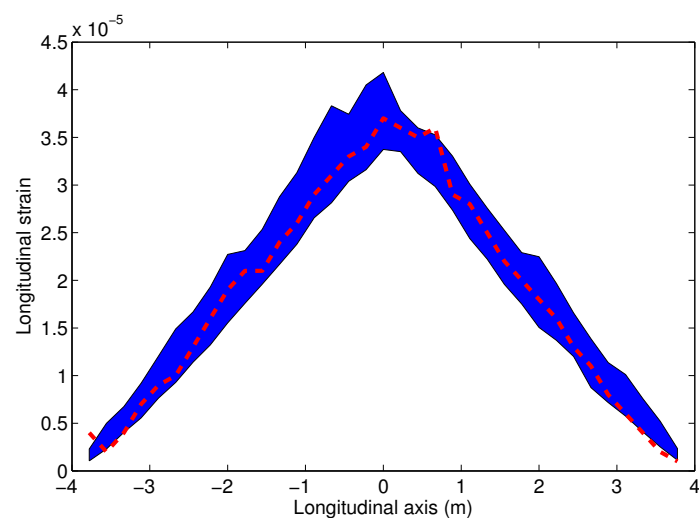

Mean strain $\pm 5 \sigma \quad-$ - - Averaged fiber optic outputs

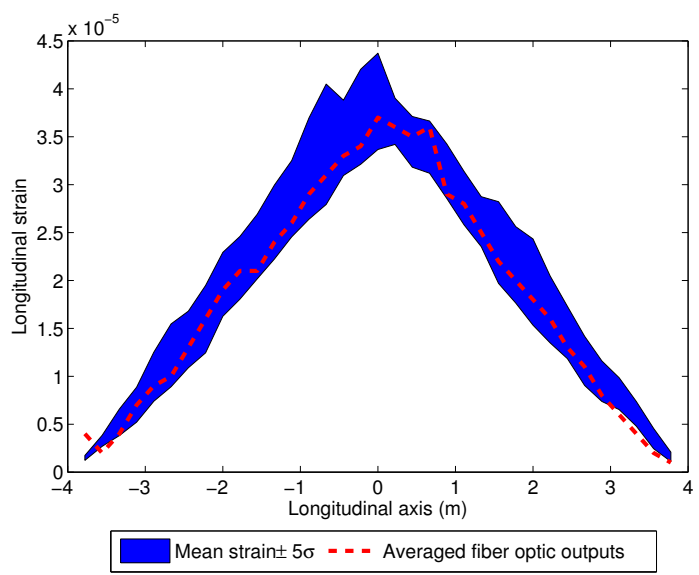

Figure 10: Five strandard deviations region of estimated strain simulated using: linear posterior (left) and quadratic posterior (right) 


\section{Conclusions and prospects}

Three model updating techniques, involving different kind of regularization, have been compared; their respective ability to localize and to quantify early

month, the model updating process can be run to actualize the cartography of Young's moduli in the structure. Hence, the site manager can follow the time evolution of the damage indicator. In case of abnormal variation, an alarm could be generated and a visual inspection could be recommended.

\section{Acknowledgments}

This research was supported by Conseil Régional d'Ile de France. This work was conducted as part of the project "SIPRIS", which involves several partners: Advitam (Vinci), ASF (Highways of the South of France), CEDRAT, ESIEEParis, NECS, SYROKKO and IFSTTAR.

\section{References}

[1] A. Rytter, Vibrational based inspection of civil engineering structures, Ph.D. thesis, University of Aalbork, Denmark (1993).

[2] K. Worden, C. R. Farrar, G. Manson, G. Park, The fundamental axioms of structural health monitoring, Proceed. R. Soc. A, Math. Phys. Eng. Sci. 463 (2082) (2007) 1639-1664.

[3] J. Brownjohn, Structural health monitoring of civil infrastructure, Proceed. R. Soc. A, Math. Phys. Eng. Sci. 365 (1851) (2007) 589-622. 
[4] A. Deraemaeker, K. Worden (Eds.), New Trends in Vibration Based Structural Health Monitoring, Springer, 2010.

[5] C. Niezrecki (Ed.), Structural Health Monitoring and Damage Detection, Volume 7: Proceedings of the 33rd IMAC, A Conference and Exposition on Structural Dynamics, 2015.

[6] A. Tarantola, Inverse problem theory and methods for model parameter estimation, SIAM, 2005.

[7] J. Mottershead, M. Friswell, Model updating in structural dynamics: a survey, Journal of Sound and Vibration 167 (1993) 347-375.

[8] M. Bonnet, A. Constantinescu, Inverse problems in elasticity, Inverse Problems 21 (2005) R1-R50.

[9] J. Beck, L. Katafygiotis, Updating models and their uncertainties. i: Bayesian statistical framework, ASCE J. Eng. Mech. 124 (4) (1998) 455461.

[10] A. Litvinenko, H. Matthies, Inverse problems and uncertainty quantification, arXiv preprint 1321.5048.

[11] H. J. Zimmerman, Fuzzy set theory and its applications, 2nd Edition, Kluwer Academic Publishers, Boston, 1991.

[12] M. Beer, Uncertain structural design based on nonlinear structural analysis, Z. Angew. Math. Mech. 84 (2004) 740-753.

[13] D. Degrauwe, Uncertainty propagation in structural analysis by fuzzy numbers, Ph.D. thesis, Department of Civil Engineering, KU Leuven (2007).

[14] H. Guo, Z. Li, Structural damage identification based on bayesian theory and improved immune genetic algorithm, Expert Systems with Applications 39 (7) (2012) $6426-6434$. 
[15] C. Papadimitriou, E. Ntotsios, Bayesian methodology for structural damage identification and reliability assessmen, in: G. C. et al. (Ed.), Proceedings of the International Operational Modal Analysis Conference (IOMAC 2009), 2009.

[16] C. A. Riveros Jerez, E. F. A. GarcÃa Aristiz $\tilde{A}_{j}$ bal, J. E. Rivero Jerez, A comparative study of sensor placement techniques for structural damage detection, Revista EIA (2013) 23 - 37.

[17] E. Simoen, , G. De Roeck, G. Lombaert, Dealing with uncertainty in model updating for damage assessment: A review, Mechanical Systems and Signal Processing 56-57 (2015) 123-149.

[18] B. Rosic, A. Kucerova, J. Sykora, O. Pajonk, A. Litvinenko, H. Matthies, Parameter identification in a probabilistic setting, Engineering Structures 50 (2013) 179-196.

[19] A. Tikhonov, Y. Arsenin, Solutions to ill-posed problems, Wintson-Widley, New York, 1977.

[20] P.-E. Charbonnel, P. Ladevèze, F. Louf, A robust CRE-based approach for model updating using in situ measurements, Computers \& Structures 129 (2013) 63-73.

[21] J. Waeytens, V. Le Corvec, P. Lévèque, D. Siegert, F. Bourquin, Elastodynamics model updating for the monitoring of reinforced concrete beam: methodology and numerical implementation, Applied Mechanics and Materials 513-517 (2014) 3401-3406.

[22] L. Chamoin, P. Ladevèze, J. Waeytens, Goal-oriented updating of mechanical models using the adjoint framework, Computational Mechanics 54 (2014) 1415-1430.

[23] M. Bonnet, W. Aquino, Three-dimensional transient elastodynamic inversion using an error in constitutive relation functional, Inverse Problems 31 (3) (2015) 035010. 
[24] J. Mazars, A description of micro- and macroscale damage of concrete structures, Engineering Fracture Mechanics 25 (1986) 729-737.

[25] V. Morozov, On the solution of functional equations by the method of regularization, Soviet Math. Dokl. 7 (1966) 414-417.

[26] P. Ladeveze, D. Leguillon, Error estimate procedure in the finite-element method, SIAM Journal on Numerical Analysis 20 (1983) 485-509.

[27] P. Ladevèze, M. Reynier, A localization method of stiffness errors for the adjustment of FE models, ASME publishers, 1989, Ch. FE Modeling and Analysis, pp. 355-361.

[28] P. Ladeveze, A. Chouaki, Application of a posteriori error estimation for structural model updating, Inverse problems 15 (1999) 49-58.

[29] S. M. Schennach, Y. Hu, Nonparametric identification and semiparametric estimation of classical measurement error models without side information, Journal of the American Statistical Association 108 (501) (2013) 177-186.

[30] J. A. Goulet, P. Kripakaran, I. F. C. Smith, Estimation of modelling errors in structural system identification, in: Proceedings of 4th International Conference on Structural Health Monitoring of Intelligent Infrastructure, 2009 , p. 359. 